\title{
Multi-label classification based ensemble learning for human activity recognition in smart home
}

\begin{abstract}
In recent times with advancements in wireless sensor technologies, human activity recognition in smart home environments have gained significant interest amongst the researchers. Numerous literatures have been focusing only on single occupant-based activity recognition because of escalating difficulties with multiple occupants within the same conditions. But having multiple occupants is more general. Therefore, in this study, the application of the Classifier Chain method of Multi-Label Classification is described to address the complicated problem of multi-resident activity recognition. Four different classifiers namely Bernoulli Naïve Bayes, Decision Tree, Logistic Regression and K-Nearest Neighbor as base classifiers for this multi-label classification approach are implemented and a comparative study of these model's performance is presented. Furthermore, the Majority Voting Ensemble Classifier method based on the ensemble learning is developed for the activity recognition problem. All these models are evaluated using several evaluation metrics on publicly available ARAS datasets. Through the results obtained from experiments, it can be deduced that the Classifier Chain method not only handles the challenges of this complex problem competently but also exhibits the importance of multi-label classification approach towards the field of activity recognition.
\end{abstract}

Keyword: Activity recognition; Classifier chain; Ensemble learning; Multi-label classification; Smart home 